\title{
Editorial
}

\section{Comentarios sobre el Informe de labores del Ministerio de Salud durante el año 2007}

Después de leer el informe de las funciones desempeñadas por el Ministerio de Salud durante el 2007 presentado por la Dra. María Luisa Ávila creo necesario escribir un comentario sobre el mismo, para que los médicos del país conozcan, los puntos relevantes del mismo y despierte en ellos el interés de leerlo completo.

La disminución del 50\% de las muertes maternas con respecto al año 2006 es el logro más importante de este año. Bien sabido es que la maternidad constituye en la mayoría de las veces una experiencia positiva y satisfactoria, en algunas ocasiones se convierte en una experiencia de muerte. Actualmente la mortalidad materna constituye un problema de salud pública a nivel mundial por lo que se ha propuesto la meta de disminuir en un $24 \%$ su mortalidad previsible, en el próximo cuatrienio.

La siguiente figura tomada del informe muestra con claridad la disminución que hemos tenido.

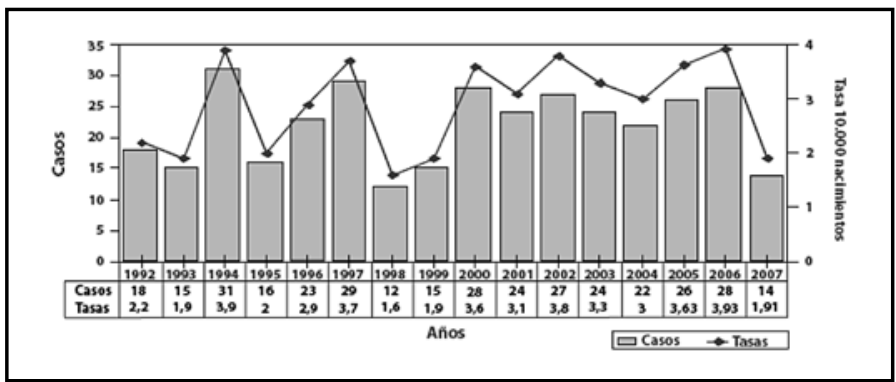

Figura 1. Tendencia de la mortalidad materna según año. Costa Rica, 1992-2007 (tasa/10.000 nacimientos).

Con lo que no solamente cumplimos con las metas acordadas para el cuatrienio, sino que nos colocamos en primer lugar en Latinoamérica como se muestra en el siguiente cuadro.

\begin{tabular}{|lcc|}
\hline \multicolumn{3}{|c|}{$\begin{array}{c}\text { Cuadro 1. Mortalidad materna en cinco países } \\
\text { latinoamericanos. Ministerio de Salud, 2007 }\end{array}$} \\
\hline País & $\begin{array}{c}\text { Número de } \\
\text { muertos }\end{array}$ & $\begin{array}{c}\text { Tasa/100.000 } \\
\text { nacimientos }\end{array}$ \\
\hline Costa Rica & 14 & 19,1 \\
Chile & 50 & 19,8 \\
Cuba & 57 & 49,4 \\
Nicaragua & 121 & 86,5 \\
Guatemala & 670 & 148,8 \\
\hline Fuente: Situación de Salud en las Américas. Indicadores básicos \\
2007, OPS/OMS.
\end{tabular}

ISSN 0001-6002/2008/50/4/194-196 Acta Médica Costarricense, (O2008 Colegio de Médicos y Cirujanos 


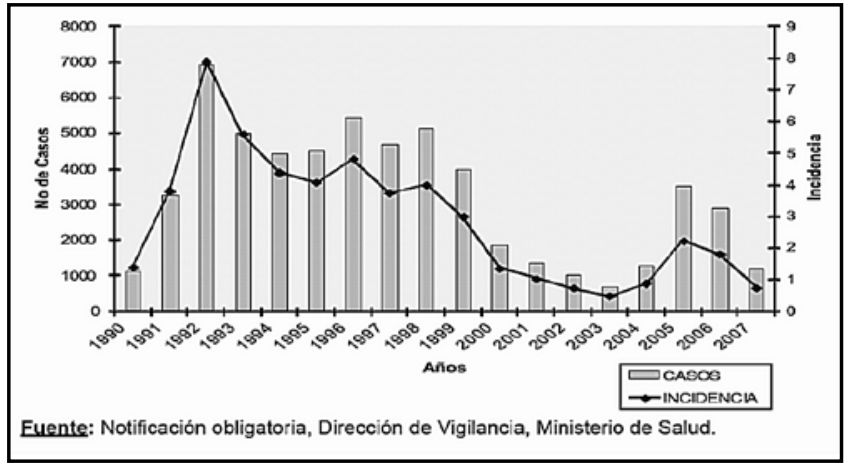

Figura 2. Casos de malaria y tasa de incidencia anual. Costa Rica, 1990-2007 (Tasa de incidencia (IPA)/1000).

También se ha presentado una disminución del dengue en las primeras 15 semanas epidemiológicas, de un 58,3\%. Es un logro importante la inclusión de la vacuna de la varicela en el esquema básico de vacunación del sector público.

El comportamiento de la tosferina en nuestro país y la relación que ha tenido con las medidas de salud aplicadas para controlarla se encuentra bien explicado en el siguiente gráfico que es importante conocer.

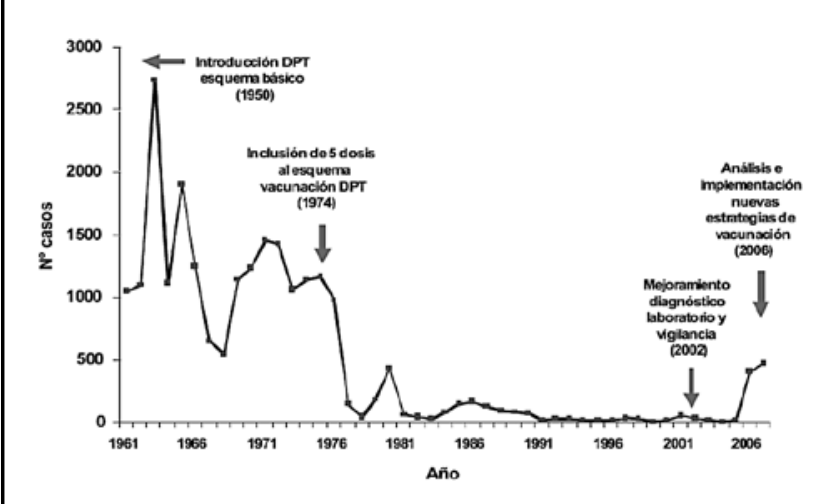

Fuente: Notificación obligatoria, Dirección de Vigilancia, Ministerio de Salud.

Figura 3. Casos reportados de tosferina. Costa Rica. 1961-2007.

En el año 2006 y en el 2007 se detecta un brote de esta enfermedad que llegó a producir algunas muertes en niños menores de seis meses en las provincias de: Heredia, Cartago y Alajuela. Ante estos hechos el Ministerio de Salud aumentó las estrategias de divulgación y actualizó las normas de tratamiento de la enfermedad, implementando la "estrategia capullo" que consistió en la vacunación de las madres en el post parto inmediato con la Tadp (tos ferina acelular difteria y tétanos), lo que impidió que actuaran como portadoras de la enfermedad y la trasmitieran a sus hijos. En el 2007 esta campaña se extendió a todo el país, lo que provoca un descenso en el número de casos de la enfermedad.
Otro aspecto importante del informe es la disminución de los casos de diarrea como causa de muerte y el cambio que ha tenido esta enfermedad en relación con la edad. Actualmente las muertes se producen en los adultos mayores, siendo los mayores de 75 años lo que presentan la cifra más alta.

En el informe se encuentran los cuadros y gráficos mejor diseñados y actualizados de la morbimortalidad de país, algo que todos los médicos deben conocer y que es fácil de accesar, en la página web del ministerio de salud, donde hay un PDF con el informe completo.

De importancia para todo médico que ejerza en el país es el conocimiento de los principales indicadores de salud que se muestran en el siguiente cuadro.

\begin{tabular}{|lccc|}
\hline \multicolumn{4}{|c|}{ Cuadro 2. Indicadores demográficos. } \\
Costa Rica, 2007 \\
\hline Indicador & Tasa & Hombres & Mujeres \\
\hline $\begin{array}{l}\text { Esperanza de vida } \\
\text { Crecimiento natural }\end{array}$ & 79 años & 76,6 años & 81,6 años \\
Tasa bruta de natalidad & 12,53 & & \\
$\begin{array}{l}\text { Tasa global de fecundidad } \\
\text { por 1000 habitantes }\end{array}$ & $1,34 / 1.000$ & $51,23 \%$ & $48,77 \%$ \\
$\begin{array}{l}\text { Tasa de reproducción bruta } \\
\text { Tasa de mortalidad general }\end{array}$ & $0,932 /$ mujer & & \\
por 1000 habitantes & 3,81 & 4,3 & 3,26 \\
$\begin{array}{l}\text { Tasa de mortalidad infantil } \\
\text { por } 1000 \text { habitantes }\end{array}$ & 10,05 & 11,13 & 8,91 \\
$\begin{array}{l}\text { Tasa de mortalidad materna } \\
\text { por } 10.000 \text { nacimientos }\end{array}$ & 1,91 & & \\
\hline
\end{tabular}

El esfuerzo que se ha hecho en el fortalecimiento de la gestión institucional mediante la ejecución del proyecto de Desarrollo Organizacional es lo que ha permitido un continuo avance en la función, tanto para los clientes externos como los internos.

Algunos hechos merecen mencionarse como son: el cierre del botadero de Río Azul, que había estado intentándose por años sin que se lograra realizarlo. No solo se cerró, sino que se están haciendo los pasos necesarios para que el sitio, en lugar de perjudicar a la comunidad, sea una zona agradable.

También es importante mencionar las acciones realizadas en protección de la salud de la comunidad, como son el cierre de Hoteles que incumplen y las acciones tomadas en algunos restaurantes de comidas en San José. En ambos casos, al no cumplir con los patrones de higiene, los primeros al verter aguas contaminadas a la playa y los segundos por contaminación de los alimentos, fueron cerrados. 
Llama la atención la persistencia de la desproporción en la asignación de recursos entre las distintas instituciones de salud del país, el informe presenta un gráfico donde se muestran los recursos que gastaron cada una de estas instituciones desde el ano 2003 al 2007. Podemos ver como el Ministerio de Salud tiene un presupuesto muy inferior al de la CCSS y el aumento que ha tenido cada año es insignificante. Otro aspecto que es necesario comentar es el aumento en los gastos de la CCSS el cual es proporcionalmente mayor que el de las otras instituciones, y a pesar de que la comparación no es válida, porque tienen acciones distintas, que no permiten compararse, si debe ponérsele atención. Probablemente, la gran inversión que se ha hecho en la compra de equipo puede ser un justificante.

Para finalizar creo que el ministerio al tener la información tan bien documentada sobre nuestra morbimortalidad podrá hacerse sentir con más peso a la hora de ser el rector de la salud de nuestro país. Este también es un logro que debe reconocerse y que deberían imitar otras instituciones.

Carlos Arrea Baixench

Miembro de Número

Academia Nacional de Medicina

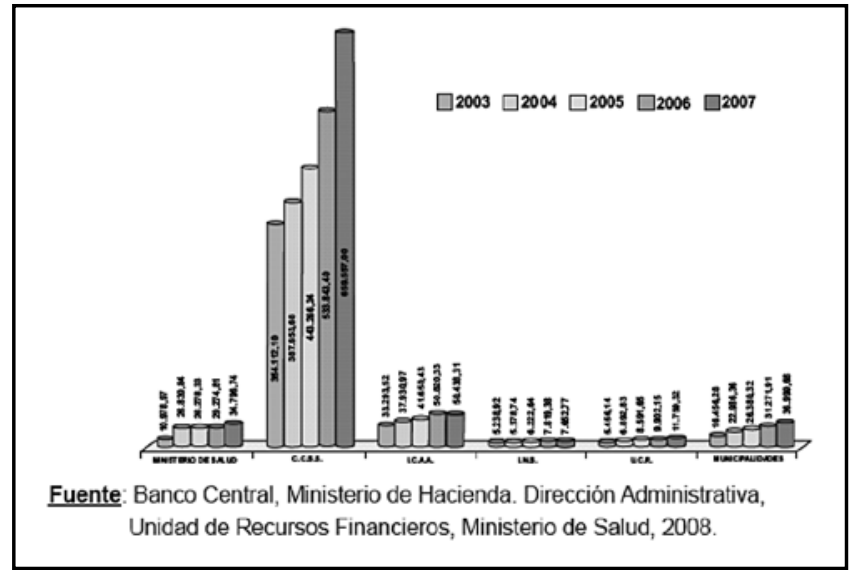

Figura 3. Gasto en el último quinquenio del Sector Salud. Ministerio de Salud y sus Instituciones. 2003-2007 (en nmillones de colones) 